\title{
Bombus gerstaeckeri MORAWITZ 1881 (Hymenoptera, Apidae) : observations sur la biologie d'un bourdon localisé et oligolectique
}

\author{
Par Simon Dellicour *, Nicolas J. Vereecken * et Denis MıCHEZ **
}

\begin{abstract}
Résumé. Les bourdons sont connus pour être majoritairement généralistes dans leurs choix floraux mais quelques espèces sont spécialisées d'un point de vue alimentaire (i.e., oligolectiques). Une étude phylogéographique visant à analyser l'évolution de l'oligolectisme au sein du sous-genre Megabombus est en cours. Le lancement de cette étude nécessitait un échantillonnage des deux espèces oligolectiques, Bombus consobrinus DAHLBORN 1832 et Bombus gerstaeckeri MORAWITZ 1881, sur l'ensemble de leur aire de distribution, ce qui nous a mené à organiser plusieurs expéditions lors des étés 2010 et 2011. Nous présentons ici nos dernières observations de terrain de $B$. gerstaeckeri, espèce particulière par son caractère très localisé et sa spécialisation alimentaire sur les espèces du genre Aconitum (Ranunculaceae).
\end{abstract}

Abstract. Bombus gerstaeckeri MORAWITZ 1881 is a particular species for its scarcity and oligolecty on species of the genus Aconitum (Ranunculaceae). We performed a sampling of B. gerstaeckeri across its entire distribution range during the summers of 2010 and 2011, in the framework of a study on the evolution of floral specialisation with a phylogeographic approach in the subgenus Megabombus. Here we present recent observations during our field surveys.

Mots-clés. Apidae, Megabombus, Bombus gerstaeckeri, oligolectisme, Aconitum.

\section{Introduction}

Au sein du genre Bombus (Hymenoptera, Apidae), seules trois espèces sont qualifiées d'oligolectiques (spécialistes alimentaires) : deux Megabombus, à savoir $B$. consobrinus DAHLBOM 1832 et B. gerstaeckeri MORAWITZ 1882, spécialistes sur le genre Aconitum (Ranunculaceae) (Pittioni 1937; Löken 1961; Löken 1973; Delmas 1976; Rasmont 1988) mais aussi B. brodmannicus delmasi TKALCU 1973, une des deux sous-espèces de $B$. brodmannicus VOGT 1909 dans le sous-genre Pyrobombus, qui présente également un certain degré d'oligolectisme sur le genre Cerinthe (Boraginaceae) (Delmas 1962; Tkalců 1973; Delmas 1976; Dellicour et al. 2012 dans ce numéro d'OSMIA). Etant donné leur ressemblance morphologique et leur spécialisation alimentaire sur Aconitum, on a longtemps pensé que $B$. consobrinus et $B$. gerstaeckeri devaient être deux espèces sœurs voir même deux sous-espèces de la même espèce. La publication de la phylogénie du genre Bombus (Cameron et al. 2007) a démontré que ces deux espèces de Megabombus n'étaient pas sœurs et que l'événement évolutif de spécialisation alimentaire sur le genre Aconitum a donc eu lieu deux fois indépendamment. Les espèces sœurs de $B$. consobrinus et $B$. gerstaeckeri sont respectivement $B$. koreanus (SKORIKOV 1933) et B. supremus MORAWITZ 1887, deux Megabombus aux distributions orientales. Alors que $B$. consobrinus présente une distribution complètement paléarctique allant de la Norvège au Japon (Løken 1973), B. gerstaeckeri présente quant à lui une distribution

\footnotetext{
* Evolutionary Biology \& Ecology, Université Libre de Bruxelles, CP 160/12, Av. F.D. Roosevelt 50, B-1050 Bruxelles, Belgique. E-mail: simon.dellicour@ulb.ac.be et nicolas.vereecken@ulb.ac.be

** Laboratoire de Zoologie, Université de Mons, Place du Parc 20,

B-7000 Mons, Belgique. E-mail: denis.michez@umons.ac.be.
}

ouest-paléarctique. On le retrouve dans les Pyrénées, les Alpes, les Carpates et il serait également présent dans le Caucase (Løken 1973; Ponchau et al. 2006; Konovalova 2007). Dans les Pyrénées et les Carpates ukrainiennes, les populations sont isolées et constituées d'un petit nombre d'individus (Ponchau et al. 2006; Konovalova 2007). Quatre observations dans les Carpates roumaines ont également été répertoriées (Moczar 1953).

Dans les Alpes et les Pyrénées, B. gertsateckeri visite essentiellement les espèces d'Aconitum suivantes : $A$. anthora L. 1753, A. napellus L. 1753 et A. lycoctonum (Figure 1). Plusieurs autres plantes hôtes sont occasionnellement visitées par cette espèce, comme par exemple Epilobium angustifolium (L.) ou encore Delphinium dubium (ROUY \& FOUCAUd) PAWL. (Mahé 2008), probablement pour le nectar. Dans cet article, nous présentons un compte-rendu des dernières observations réalisées au cours de nos expéditions en 2010 et 2011.

\section{Observations récentes et distribution géographique}

Nous nous sommes rendus en 2010 sur les quatre localités décrites dans les Carpates roumaines mais n'y avons observé aucune population d'aconits. Les biotopes ne correspondaient d'ailleurs pas aux zones dans lesquelles on serait habitué à trouver ces espèces (clairières ensoleillées en montagne). La présence de $B$. gerstaeckeri dans les Carpates roumaines peut très clairement être remise en doute, d'autant plus qu'à la suite des observations de Moczar (1953), aucune autre observation n'y a été recensée. 

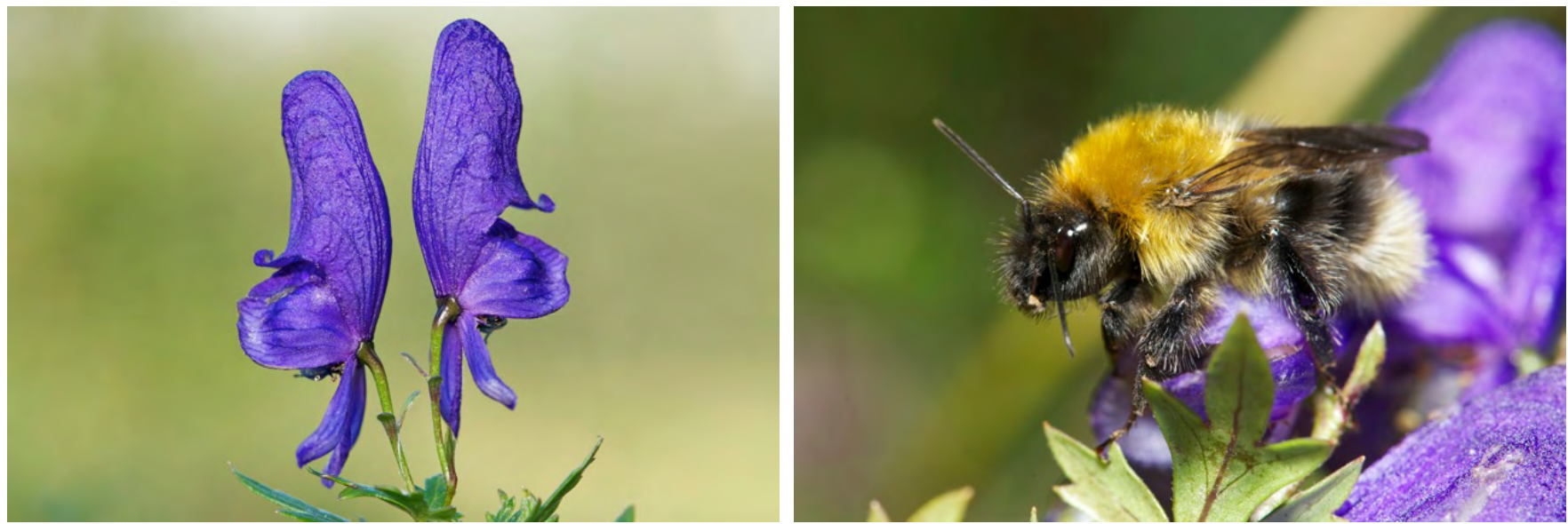

Figure 1. Détail floral d'Aconitum napellus L. 1753 (Ranunculaceae) (gauche) et mâle de Bombus gerstaeckeri Morawitz 1881 sur A. napellus (droite) (Photos NJ Vereecken)

En 2010 et 2011, nous avons observé B. gerstaeckeri sur 8 sites différents dans les Pyrénées, les Alpes et les Carpates ukrainiennes (Figure 2) :

- Gavarnie (42.696667º N, 0.017500º, WGS84), Pyrénées, France : observations d'ouvrières et de reines sur $A$. napellus, $A$. lycoctonum et $A$. anthora (31.viii.10, 16h);

- Egat $\left(42.507200^{\circ} \mathrm{N}, 2.018611^{\circ} \mathrm{E}, \mathrm{WGS} 84\right)$, Pyrénées, France : observations d'ouvrières et de reines sur $A$. napellus (29.viii.10, 12h);

- Saint-Véran $\left(44.697500^{\circ} \mathrm{N}, 5.858889^{\circ} \mathrm{E}\right.$, WGS84), Alpes, France : observations d'ouvrières et de reines sur $A$. napellus et $A$. lycoctonum (07.viii. 10, 15h) (Figure 1);

- Kandersteg $\left(46.493611^{\circ} \mathrm{N}, 7.672778^{\circ} \mathrm{E}, \mathrm{WGS} 84\right)$, Alpes, Suisse : observations de mâles sur $A$. napellus (06.ix.11, 18h)

- Vals $\left(46.618056^{\circ} \mathrm{N}, 9.188333^{\circ} \mathrm{E}\right.$, WGS84), Alpes, Suisse : observations de mâles sur $A$. napellus (07.ix.11, 14h) (Figure 1).

- Gramais $\left(47.270833^{\circ} \mathrm{N}, 10.537778^{\circ} \mathrm{E}\right.$, WGS84), Alpes, Autriche $\left(47.270833^{\circ} \mathrm{N}, 10.537778^{\circ} \mathrm{E}\right.$,
WGS84) : observations d'ouvrières et de reines $A$. napellus (28.viii.10, 14h)

- Bohinjsko Jezero $\left(46.284722^{\circ} \mathrm{N}, 13.780556^{\circ} \mathrm{E}\right.$, WGS84), Alpes, Slovénie : observations d'ouvrières et de reines sur A. lycoctonum (02.viii. $10,14 \mathrm{~h}$.

- $20 \mathrm{~km}$ SW from Vorokhta $\left(48.147500^{\circ} \mathrm{N}\right.$, $24.545833^{\circ} \mathrm{E}$, WGS84), $1470 \mathrm{~m}$, Carpates, Ukraine : observations de mâles sur $A$. variegatum (21.viii.11, 16h).

Etant donné la rareté de cette espèce, nous avons essayé d'éviter au maximum de nuire aux populations par l'échantillonnage. Ainsi, une grande partie de l'étude génétique se fera sur base d'extractions d'ADN réalisées à partir d'une patte prélevée sur des individus de collection. Pour les populations dont nous ne possédions pas ou peu d'individus de collection, nous n'avons échantillonné que des ouvrières ou des mâles en fin de phénologie (p.ex. encore pour les deux populations échantillonnées en Suisse). Sur les différents sites, nous n'avons jamais observé de visites de $B$. gerstaeckeri sur des plantes autres que des aconits.

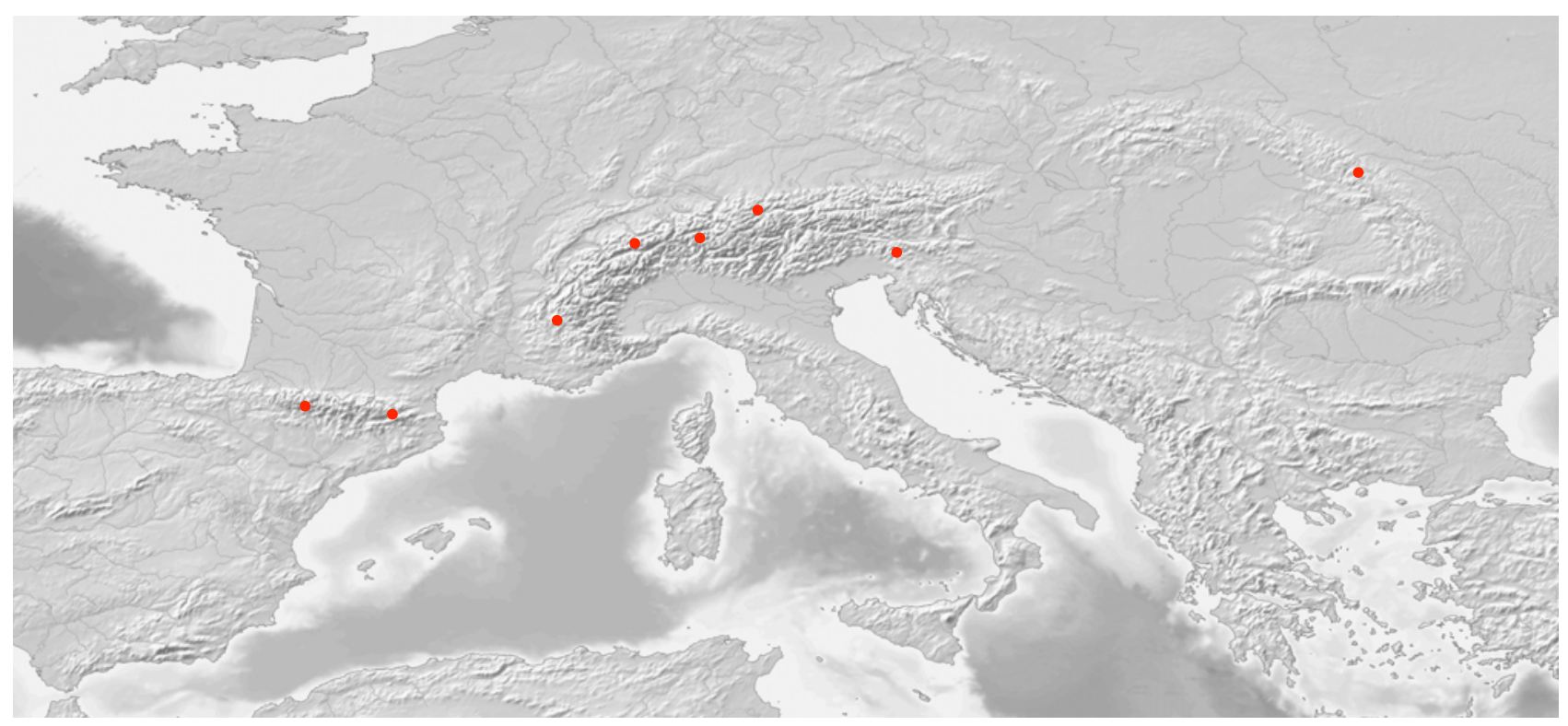

Figure 2. Carte d'échantillonnage de Bombus gerstaeckeri MORAWITZ 1881 au cours des étés 2010 et 2011. 


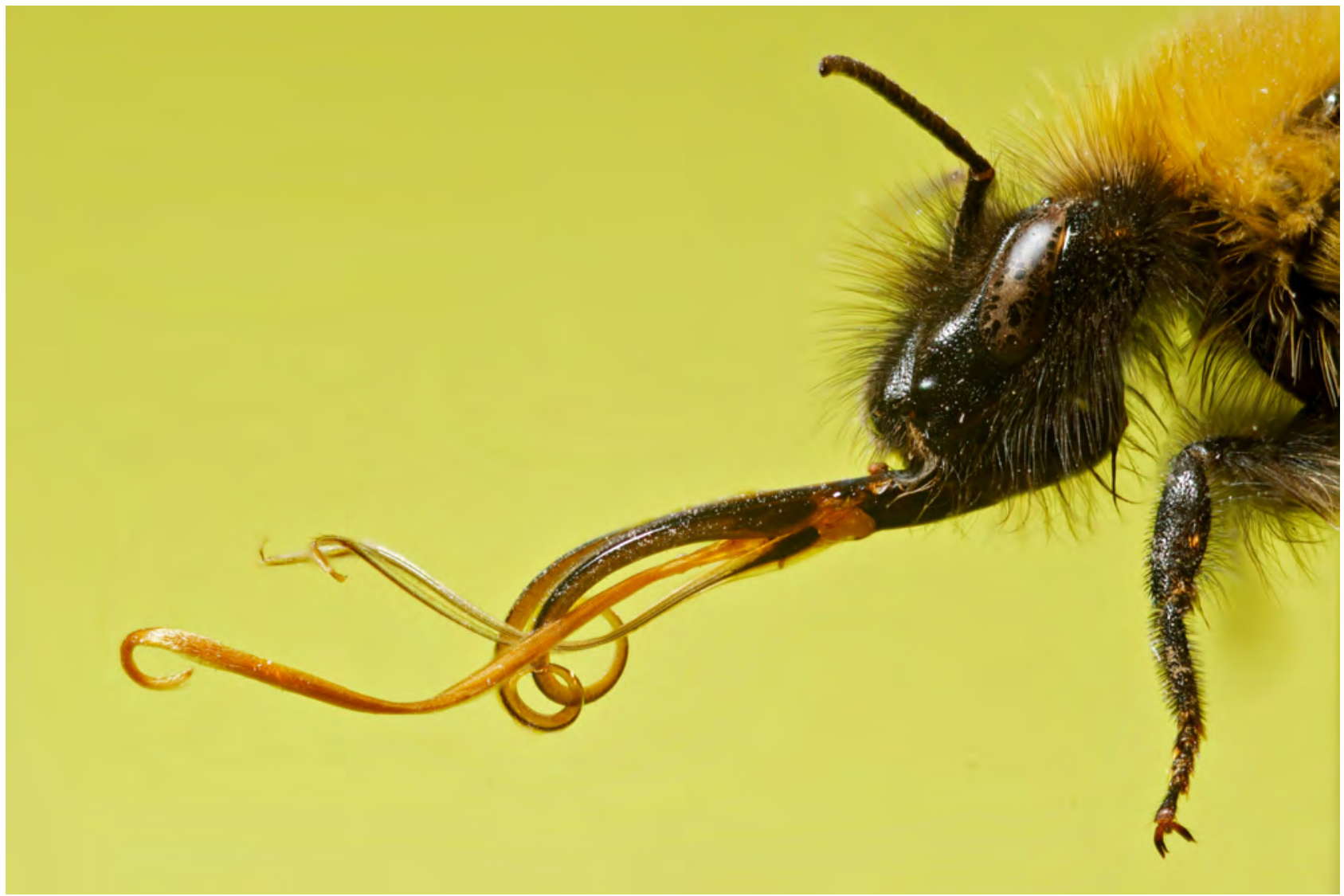

Figure 3. Détail du long proboscis et de la tête allongée de Bombus gerstaeckeri MoRAWITZ 1881, typique du sous-genre Megabombus (Photo NJ Vereecken)

Bombus gerstaeckeri n'est bien entendu pas le seul bourdon à visiter les aconits. Sur les différents sites, nous y avons entre autres également observé beaucoup de visites de $B$. hortorum (L. 1761), mais aussi de $B$. wurflenii RADOSZKOWSKI 1860. Mais aucune abeille solitaire n'a été observée. Comme détaillé par Ponchau et al. (2006), B. gerstaeckeri serait avantagé par un proboscis notablement long (Figure 3) qui lui permettrait d'atteindre et collecter plus de nectar dans ces fleurs que ses concurrents. Apparu deux fois de manière indépendante, l'oligolectisme sur le genre Aconitum peut très clairement être interprété sous l'hypothèse d'une stratégie alimentaire basée sur une spécialisation sur des ressources abondantes, riches en pollen/nectar et faiblement exploitées.

\section{Remerciements}

Nous tenons tout d'abord à remercier le FNRS qui finance le projet de recherche global sur l'étude de la spécialisation alimentaire chez les abeilles (FRFC 2.4613.10). Merci à T. Lecocq, A. Jenic, P. Lhomme, A. Konovalova, V.G. Radchenko, C. Thonar et H. Vanderschuren pour leur aide précieuse et/ou leur accueil au cours des campagnes de collecte. Merci aussi finalement à S. Iserbyt, P. Rasmont, F. Amiet et A. Müller pour leurs informations de terrain.

\section{Références bibliographiques}

Gameron SA, Hines HM, Williams PH, 2007. A comprehensive phylogeny of the bumble bees (Bombus). Biological fournal of the Linnean Society 91: 161-188.

Delmas R, 1962. Notes zoogéographiques et systématiques sur les Bombidae. I. - Le Bombus brodmannicus VOGT des Alpes françaises. Annales de l'Abeille 5(3): 175-179.
Delmas R, 1976. Contribution à l'étude de la faune française des Bombidae (Hymenoptera, Apoidea, Bombidae). Annales de la Société Entomologique de France (n.s.) 12: 247-290.

Konovalova I, 2007. The first record of the rare oligolectic bumblebee Bombus gerstaeckeri MORAWITZ (Hymenoptera: Apidae: Bombini) from Ukraine. Annales de la Société Entomologique de France (n.s.) 43(4): 441-443.

Løken A, 1961. Bombus consobrinus DAHLBOM, an oligolectic bumblebee (Hymenoptera, Apidae). Proceeding of the XIth Int. Congr. Ent. 1960 1: 598-603.

Løken A, 1973. Studies on Scandinavian Bumblebees (Hymenoptera, Apidae). Norsk Entomologisk Tidsskrift 20: 1-218.

Mahé G, 2008. Bourdons rares du Parc Naturel Régional du Queyras (Hautes-Alpes, France). OSMIA 2: 21-25.

Moczar M, 1953. Magyarország és a környezö területek dongóméheinek. (Bombus LATR.) rendszere és ökológiája, Magyar Nemzeti Múzeum. Termézettudományi Múzeum évkönyve. Annales Historico-naturales Musei Nationalis Hungarici 4: 131-159.

Pittioni B, 1937. Bestäubung und Nektarraub beim Gelben Eisenhut (Aconitum vulparia RCHB). Aus der Heimat, Stuttgart 50: 209-213.

Ponchau O, Iserbyt S, Verhaeghe JC, Rasmont P, 2006. Is the caste-ratio of the oligolectic bumblebee Bombus gerstaeckeri MORAWITZ (Hymenoptera: Apidae) biased to queens? Annales de la Société Entomologique de France 42(2): 207-214.

Tkalců B, 1973. Taxonomie von Pyrobombus brodmannicus (VOGT) (Hymenoptera, Apoidea, Bombinae). Acta entomologica Bohemoslovaca 70(4): 259-268.

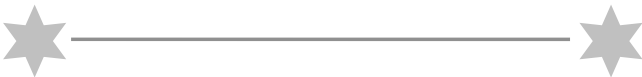

